\title{
ON EVEN ENTIRE FUNCTIONS WITH ZEROS HAVING A DENSITY
}

\author{
BY \\ R. M. REDHEFFER
}

If $F(z)$ is an entire function of order $<2$, then

$$
F(z)=a z^{m} e^{b z} \prod_{n=0}^{\infty}\left(1-z / \lambda_{n}\right) e^{z / \lambda_{n}} .
$$

When $F(z)$ is even or odd the form becomes

$$
F(z)=a z^{m} \prod_{n=0}^{\infty}\left(1-z^{2} / \lambda_{n}^{2}\right)
$$

with $m$ even or odd, respectively. Our purpose here is to study the relation between the growth of $F(z)$ in (1) and the distribution of the zeros, a topic which has already formed the subject of many investigations. Without essential loss of generality we can, and do, take

$$
F(z)=\prod_{n=0}^{\infty}\left(1-z^{2} / \lambda_{n}^{2}\right), \quad \sum 1 /\left|\lambda_{n}\right|^{2}<\infty .
$$

For simplicity it is assumed in addition that $\lambda$ 's are real, unless we state the contrary, the notation being so chosen that $0<\lambda_{0} \leqq \lambda_{1} \leqq \lambda_{2} \leqq \cdots$. Throughout the paper $\Lambda(u)$ denotes the number of $\lambda$ 's which are less than $u$. Although Levinson [1] has shown how some of the results for complex $\lambda$ 's can be deduced from those for real, the reduction of the one case to the other is difficult; and hence the assumption of real $\lambda$ 's involves a loss of generality.

Within this framework we give simple proofs, and sharpened forms, of many known results, to which reference is made at appropriate points of the sequel. Some of the facts established are used to discuss the $L^{2}$ completeness of exponentials on a given finite interval.

Representations for $F(x)$. As in [3], for real $x$

$$
\begin{aligned}
\log |F(x)| & =\sum \log \left|1-x^{2} / \lambda_{n}^{2}\right| \\
& =\int_{a}^{\infty} \log \left|1-x^{2} / u^{2}\right| d \Lambda(u) \\
& =\int_{a}^{x-b} \log \left(x^{2} / u^{2}-1\right) d \Lambda+\int_{x+b}^{\infty} \log \left(1-x^{2} / u^{2}\right) d \Lambda
\end{aligned}
$$

Presented to the Society, November 29, 1952, May 2, 1953, and September 5, 1953; received by the editors March 23, 1953. 
where $a$ is any real number between 0 and $\lambda_{0}$, while $b$ is any real number such that the interval $(x-b, x+b)$ has no zeros. Since $\sum 1 / \lambda_{n}^{2}<\infty$ for convergence of the infinite product, and since the $\lambda$ 's are monotonic, we have $n / \lambda_{n}^{2} \rightarrow 0$. From this it follows that

$$
\Lambda(u)=o\left(u^{2}\right),
$$$$
u \rightarrow \infty \text {. }
$$

If we now integrate by parts in (3), Equation (4) shows that the integrated term vanishes at $\infty$. It also vanishes at $a$, since $a<\lambda_{0}$. Combining the integrated terms at $x-a$ and $x+a$, as in [3], leads to

$$
\log |F(x)|=\left\{\int_{a}^{x-b}+\int_{x+b}^{\infty}\right\} \frac{\Lambda(u)}{u} \frac{2 x^{2} d u}{x^{2}-u^{2}}+\frac{\Lambda(x)}{x}(3 b+E)
$$

where the error-term $E$ satisfies $E=O(1 / x)$ as $x \rightarrow \infty$. It is shown in [3] that $\log |F(x)|$ is dominated by the expression on the right of (5), for large $x$, even when the interval $(x-b, x+b)$ contains zeros; that is, the extra zeros introduce a new error term which is always negative.

If we define $A(u)$ by

$$
\Lambda(u)=A(u)+D u
$$

where $D$ is constant, then (5) yields, after a short calculation,

$$
\begin{aligned}
\log |F(x)|= & \left\{\int_{a}^{x-b}+\int_{x+b}^{\infty}\right\} \frac{A(u)}{u} \frac{2 x^{2} d u}{x^{2}-u^{2}} \\
& +(3 b+E) \frac{\Lambda(x)}{x}-\left(2 a+b+E_{1}\right) D
\end{aligned}
$$

where $E_{1}$ also is $O(1 / x)$. Within terms of this order the result is independent of $a$ and $b$, as it should be.

Since $x+b<x^{2} /(x-b)<x^{2} / a<\infty$ for large $x$, and since the integrand in (5) is negative for $u>x$, the expression (5) is increased(1) if the limits $x+b, \infty$ are replaced respectively by $x^{2} /(x-b)$ and $x^{2} / a$. Making that change in (5), and letting $u=v x$ in the first integral, $u=x / v$ in the second, gives

$$
\begin{aligned}
(1 / x) \log |F(x)| & \leqq \int_{a / x}^{1-b / x} p(v, x) 2 d v /\left(1-v^{2}\right)+o(1), \\
p(v, x) & =\frac{\Lambda(v x)}{v x}-\frac{\Lambda(x / v)}{x / v}
\end{aligned}
$$

as in [3]. That the inequality holds for all $x$ follows from the remark made earlier concerning the effect of zeros near $x$. Also the relation holds as an equality, with $o(1)$ replaced by $O(1 / x)$, when $\Lambda(u) / u$ is bounded and $\left|x-\lambda_{n}\right|$ $>c>0$.

(1) This remark, due to Robert Steinberg, simplifies the original presentation. 
A theorem of Carlson. The condition $\lim \Lambda(u) / u=D$ implies $\log |F(x)|$ $\leqq o(x)$, provided $\lambda_{n+1}-\lambda_{n}>c>0$ (see [4]). Although previous proofs $[1 ; 3 ; 4]$ make essential use of the latter hypothesis, it has been observed (for example by Levinson [1]) that the condition can be weakened. Later it was shown by Pfluger [7] that the condition can be dropped altogether; in fact, one requires only $\sum \operatorname{Im}\left(1 / \lambda_{n}\right)<\infty$. For even functions a similar result is deducible from the work of Titchmarsh [8]. Here we give a new proof which uses realvariable methods only:

Theorem I (Titchmarsh, Pfluger). If $\lim \Lambda(u) / u=D$, then $\log |F(x)|$ $\leqq o(x)$.

For proof, write the integral in (8) as

$$
\left\{\int_{a / x}^{\delta}+\int_{\delta}^{1-\delta}+\int_{1-\delta}^{1-b / x}\right\} p(v, x) 2 d v /\left(1-v^{2}\right)
$$

where $\delta$ is a fixed small number. As in [3] the first integral is $O(\delta)$, since $\Lambda(u) / u$ is bounded. Again as in [3], the second integral tends to zero, for fixed positive $\delta$, when $x \rightarrow \infty$. The third integral may be written

$$
\begin{aligned}
& \int_{1-\delta}^{1-b / x} \frac{\Lambda(v x)-\Lambda(x / v)}{x} \frac{d v}{1-v^{2}} \\
+ & \int_{1-\delta}^{1-b / x}\left[\frac{\Lambda(v x)}{v x}+\frac{\Lambda(x / v)}{v(x / v)}\right] \frac{d v}{1+v} .
\end{aligned}
$$

The expression (10) is $\leqq 0$ since $\Lambda(u)$ is monotonic, and (11) is $O(\delta)$ since $\Lambda(u) / u$ is bounded. Choosing $\delta$ first and then letting $x \rightarrow \infty$, we obtain the theorem.

Since $\Lambda(u)$ is nondecreasing, it is a simple exercise (probably due to Landau; also see the lemma for Theorem XI) to show that

$$
\lim _{u \rightarrow \infty} \frac{1}{u} \int_{0}^{u} \frac{\Lambda(t)}{t} d t=D
$$

implies $\lim \Lambda(u) / u=D$ and hence implies Theorem I. Thus, Cesàro existence of the limit suffices. The Littlewood $O(1 / n)$ theorem shows that Poisson summation

$$
\lim _{x \rightarrow 1-}(1-x) \sum\left(\lambda_{n} / n\right) x^{n}=D
$$

also suffices, provided $\lambda_{n}=O(n)$. A less trivial extension of Theorem $I$ is possible, however, as we shall now see. The only use made of the condition $\lim \Lambda(u) / u=D$ was to obtain the boundedness of $\Lambda(u) / u$, and to ensure the vanishing of $\int_{\delta}^{1-\delta}$ in (9) as $x \rightarrow \infty$. In fact the proof shows that $\log ^{+}|F(x)|$ $=o(x)$ if $\Lambda(u) / u<M$, and 


$$
\lim _{\delta \rightarrow 0} \lim _{x \rightarrow \infty} \int_{\delta}^{1-\delta} p(v, x) d v /\left(1-v^{2}\right)=0 .
$$

It will be seen later that this result and the following $\left({ }^{2}\right)$ generalize Theorem I, in that they apply even when $\Lambda(u) / u$ has no limit.

THEOREM II. If $\Lambda(u) / u$ is bounded and if, for each positive $v$,

$$
\lim _{x \rightarrow \infty}\left[\frac{\Lambda(v x)}{v x}-\frac{\Lambda(x / v)}{x / v}\right]=0,
$$

then

$$
\lim _{x \rightarrow \infty} \log ^{+}|F(x)| / x=0 .
$$

It suffices to show that the limit (13) holds uniformly in $v$, if $0<\delta \leqq v$ $\leqq 1-\delta$; for from this (12) follows. The uniformity is a simple consequence of

Lemмa 1. Under the hypothesis of Theorem II we have

$$
\lim \frac{\Lambda\left(a_{i} x_{i}\right)-\Lambda\left(x_{i}\right)}{x_{i}}=0, \quad i \rightarrow \infty,
$$

whenever $\lim a_{i}=1, \lim x_{i}=\infty$.

Let us consider the $i$ 's in (14) for which $a_{i} \geqq 1$; discussion of the others is similar. Given $\epsilon>0$, choose $a>1$ so that $(a-1) M<\epsilon$, where $M$ is a bound for $\Lambda(u) / u$. If $i$ is so large that $1 \leqq a_{i} \leqq a$, the monotonic character of $\Lambda(u)$ gives

$$
\begin{aligned}
0 & \leqq \frac{\Lambda\left(a_{i} x_{i}\right)-\Lambda\left(x_{i}\right)}{x_{i}} \leqq \frac{\Lambda\left(a x_{i}\right)}{x_{i}}-\Lambda\left(x_{i}\right) \\
& =\left[\frac{\Lambda\left(a x_{i}\right)}{a x_{i}}-\frac{\Lambda\left(x_{i}\right)}{x_{i}}\right]+\frac{\Lambda\left(a x_{i}\right)}{a x_{i}}(a-1) .
\end{aligned}
$$

As $x_{i} \rightarrow \infty$ the expression in brackets approaches zero, in view of (13); and hence, by our choice of $a$, the limit is at most $\epsilon$.

Having established the lemma, we suppose now that (13) holds, but that the convergence is not uniform for $0<\delta \leqq v \leqq 1-\delta$. This means that there is a sequence $v_{i}$ taken from $[\delta, 1-\delta]$, and a sequence $x_{i} \rightarrow \infty$, such that

$$
\left|\frac{\Lambda\left(x_{i} / v_{i}\right)}{x_{i} / v_{i}}-\frac{\Lambda\left(x_{i} v_{i}\right)}{x_{i} v_{i}}\right| \geqq p>0,
$$

where $p$ is fixed. The Weierstrass theorem enables us to assume $v_{i} \rightarrow v$, where $\delta \leqq v \leqq 1-\delta$.

(2) It is a pleasure to acknowledge the author's indebtedness to Paul Erdös, who pointed out the possibility of extending Theorem I in this way, and also verbally sketched a proof. 
Equation (13) for this value $v$ yields

$$
\lim \left|\frac{\Lambda\left(x_{i} / v_{i}\right)}{x_{i} / v_{i}}-\frac{\Lambda\left(x_{i} v^{2} / v_{i}\right)}{x_{i} v^{2} / v_{i}}\right|=0,
$$$$
x_{i} \rightarrow \infty \text {, }
$$

the role of $x$ in (13) being taken by $x_{i} v / v_{i}$. Combining (16) with (15) shows that

$$
\left|\frac{\Lambda\left(x_{i} v^{2} / v_{i}\right)}{x_{i} v^{2} / v_{i}}-\frac{\Lambda\left(x_{i} v_{i}\right)}{x_{i} v_{i}}\right| \geqq p / 2
$$

for large $x_{i}$. Since $\left[x_{i} v^{2} / v_{i}\right] /\left(x_{i} v_{i}\right)=\left(v / v_{i}\right)^{2} \rightarrow 1$, the inequality (17) contradicts the lemma.

The converse. We now enquire whether the condition $\log ^{+}|F(x)|=o(x)$ implies a regularity condition on the $\lambda_{n}$ 's. That Carlson's theorem does not admit a converse is indicated by the work of Pfluger [7]; but here we construct a counterexample which shows more specifically how far one can go:

Theorem III. Suppose given $p, q$, and $A$, with $p>q>0$ and $A<1$. Then there exists a sequence $\left\{\lambda_{n}\right\}$ satisfying $\lambda_{n+1}-\lambda_{n}>A / p, \lim \sup \Lambda(u) / u=p$, $\lim \inf \Lambda(u) / u=q$, and $\log ^{+}|F(x)|=o(x)$.

Probably the conditions $q>0, A<1$ can be replaced by $q \geqq 0, A \leqq 1$ with only minor modification of the proof. To establish III as it stands, let $p=a+b, q=a-b$, and define

$$
L(u)=u\left[a+b \cos (\log u)^{1 / 2}\right],
$$

$u>1$.

Then $\lim \sup L(u) / u=p, \lim \inf L(u) / u=q$, and $L(u)$ satisfies (13), as is seen by a short calculation. Moreover for large $u$ we have $0<L^{\prime}(u)<p / A$, and hence there is a distribution of zeros, $\Lambda(u)$, such that

$$
|\Lambda(u)-L(u)| \leqq 1,
$$

$u$ large,

and $\lambda_{n+1}-\lambda_{n}>A / p$. Since $L(u)$ satisfies (13), so does $\Lambda(u)$, and hence $\log ^{+}|F(x)|=o(x)$. It does not matter how we define $\Lambda(u)$ for small $u$, so long as $\Lambda(u)$ satisfies the requirements of the theorem. For, a finite number of factors in $F(x)$ only alters $F(x)$ by a polynomial, and this does not influence the estimate $\log ^{+}|F(x)|=o(x)$.

The following result $\left({ }^{3}\right)$ shows that the weaker regularity postulated in Theorem II also cannot be deduced from $\log ^{+}|F(x)|=o(x)$ :

Theorem IV. Given $c$, and $M>0$, there exists a sequence $\left\{\lambda_{n}\right\}$ such that $\lambda_{n+1}-\lambda_{n}>c, \Lambda(u) / u<M, \log ^{+}|F(x)|=o(x)$, and (13) is violated.

We give the proof in outline only. Let $e$ and $f$ be constants with $1>f>e$

(3) The result is unexpected. It had been conjectured by Paul Erdös and by the author that Theorem II might give a necessary and sufficient condition, at least when $\lambda_{n+1}-\lambda_{n}>c$. 
$>0$, and let $p$ be a large constant. Define a function $L(u)$ as follows: The graph of $L(u)$ is a straight line of slope 1 from $(p, e p)$ to infinity; it is a horizontal line from $(p, e p)$ to the point $(w p, e p), w<1$, where this horizontal line meets the line $y=f u$; it continues down a line of slope 1 until this line of slope 1 meets $y=e u$; and it coincides with $y=e u$ until it reaches the origin. Thus, $L(u)$ consists of connected straight line segments having slopes $e, 1,0,1$; the portion of zero slope joins the lines $y=f u$ and $y=e u$. Associated with $L(u)$ is a zero distribution $\Lambda(u),|\Lambda-L| \leqq 1$, and a function $F(x)$. In our considerations we may speak of $\Lambda$ and $L$ interchangeably.

Let $(x, e p)$ be a point on the horizontal part, so that $x=c p$, with $w<c<1$. An elementary calculation gives the principal value of (cf. Equation (5))

$$
\int_{a}^{\infty} \frac{L(u)}{u} \frac{2 x d u}{x^{2}-u^{2}} .
$$

For large $p$ it turns out that the result has the form

$$
-2(1-e) \log 2+O(1-w, 1-c) ;
$$

and the same is found for points $x$ on the line segments of slope 1 cut off by $e u, f u$. Now, as $f \rightarrow e$ we have $w \rightarrow 1$, hence $c \rightarrow 1$. Thus we can choose $f$ so close to $e$ that the integral (20) is negative, if $p$ is large, for all $x$ in the indicated range. Such a choice of $e$ and $f$ is now made (with $f>e$, of course).

It is clear from $(20)$ that $L(u)$ is weighted negatively for $u>x$, positively for $u<x$. (When $L(u)$ is interpreted as a zero-distribution $\Lambda(u)$, this remark merely reflects the fact that the factors in $|F(x)|$ are greater or less than 1 , according as $|x| \gg \lambda_{n}$ or $|x|<\lambda_{n}$.) If we choose a comparison function $L_{1}(u)$ such that $L_{1}(u) \geqq L(u)$ for $u<x$, and $L_{1}(u) \leqq L(u)$ for $u>x$, then the integral for $L_{1}$ dominates that for $L$. This remark enables us to determine at a glance the behavior of (20) for certain values of $x$. If $x$ is on the segment of slope $e$, choose $L_{1}(u)=e u$. The corresponding integral (20) is bounded $\left(F_{1}(x)\right.$ is essentially a cosine) so $F(x)$ is also. Similarly if $x$ is beyond the point where the second segment of slope 1 cuts $y=f u$, the choice $L_{1}(u)=f u$ shows that $F(x)$ is bounded.

We now modify $L(u)$. Instead of letting $L(u)$ follow the second segment of slope 1 on out to infinity, after a time we make $L(u)$ bend back, very gradually, until finally it coincides with the line $y=e u$. That this process can be carried out in such a way that (13) is preserved follows from the proof of Theorem III (cf. Equation (18)). We now follow the line $y=e u$ out to a large value of $x$. The stopping-place $x$ is so chosen that the early factors of $F(x)$, associated with the portion of $L(u)$ where $L(u) \neq e u$, have a product "nearly equal" to that which they would have had for $L(u)=e u$. This is possible, since the ratio of these partial products tends to a finite limit as $x \rightarrow \infty$. Starting at this point, we repeat the whole process just described: $L(u)$ leaves the line $y=e u$, following a line of slope 1 until it reaches $y=f u$. Then $L(u)$ 
continues horizontally to a point $p$, on $y=e u$, and now $L(u)$ follows a line of slope 1 , to return later, and gradually, to the line $y=e u$.

For a fixed $w$ and a sequence $p \rightarrow \infty$ we have $L(p) / p=e, L(w p) /(w p)=f$, so that (13) is violated. Since $L^{\prime}(u) \leqq 1$ at all times, the associated $\Lambda(u)$ has $\lambda_{n+1}-\lambda_{n} \geqq 1$. Considering $F(k x)$ gives $\lambda_{n+1}-\lambda_{n} \geqq c, \Lambda(u) / u<M$, and thus the proof is complete.

The foregoing theorems show that $\left\{\lambda_{n}\right\}$ can be rather irregular, and yet allow $\log ^{+}|F(x)|=o(x)$. Continuing in this vein we show that $\left\{\lambda_{n}\right\}$ may have large gaps, so that $F(x)$ has large intervals free of zeros, and yet $\log ^{+}|F(x)|$ $=o(x)$.

TheOREm V. If $M>0$ there is a distribution of zeros such that $\Lambda(u) / u<M$, $\lambda_{n+1}-\lambda_{n}>c, \log ^{+}|F(x)|=o(x)$, and yet $\Lambda\left(x_{i}\right)=\Lambda\left(y_{i}\right)$ for an infinite sequence $y_{i} \rightarrow \infty, x_{i} / y_{i} \rightarrow \infty$.

Let $p, w$, and $f$ be constants, with $p$ and $w$ large, and with $f$ satisfying $0<2$ wef $<1$. The role of $y_{i}$ in the theorem will be taken by a sequence $p \rightarrow \infty$, and $x_{i}$ by a sequence $p w$. Define $L(u)$ as the continuous function which follows the line $y=f u$ up to $u=p$, then is horizontal to $u=w p$, then follows a line of slope 1 to infinity. For the principal value one finds

$$
\begin{aligned}
\int_{0}^{\infty} \frac{L(u)}{u} \frac{2 x d u}{x^{2}-u^{2}} & =2 s \log s-(s+1) \log (s+1)-(s-1) \log (s-1) \\
& +f[(1+c) \log (1+c)-2 c \log c-(1-c) \log (1-c)]
\end{aligned}
$$

after a short calculation, where $c=p / x$ and $s=c w$. We assume $x$ on the horizontal portion of $L(u)$, so that $1>c>1 / w, w>s>1$. Discussion of other values of $x$ is similar, most values being dealt with at a glance by introduction of a comparison function $L_{1}(u)$.

The derivative of the terms involving $s$ is $\log s^{2} /\left(s^{2}-1\right)$, hence positive. These terms have their maximum at $s=w$, therefore, and the maximum is approximately $-1 / w$, since $w$ is large. Similarly, the coefficient of $f$ has a maximum at $2 c^{2}=1$, and the maximum does not exceed 2 . The given condition on $f$ shows now that the integral is negative for all $x$ in the given range; and actually the same is true for all large $x$.

We now modify $L(u)$. Given $w_{1}>w$, we choose $f_{1}<1 /\left(2 w_{1}\right)$. Instead of following a line of slope 1 out to infinity, $L(u)$ is bent back gradually until it coincides with the line $y=f_{1} u$. Choosing $p_{1} \gg p$, we repeat the process formerly described, and so on. The approximating $\Lambda(u)$ satisfies $\lambda_{n+1}-\lambda_{n} \geqq 1$, since $L^{\prime}(u) \leqq 1$; and considering $F(k x)$ gives the theorem.

So far the results have been negative; we have shown that various smoothness conditions on $\left\{\lambda_{n}\right\}$ are not necessary for $\log ^{+}|F(x)|=o(x)$. If $\lambda_{n+1}$ $-\lambda_{n}>c>0$, however, it turns out that (12) is necessary. (We already know that (12) is sufficient even under the lighter hypothesis $\Lambda(u) / u<M$.) Necessity results from the following: 
Theorem VI. If $\lambda_{n+1}-\lambda_{n}>c>0$, then $\log ^{+}|F(x)|=o(x)$ implies (12).

The proof is actually contained in [3], where it is shown that $\int_{1-\delta}^{1-b / x}$ in (9) is small when $\delta$ is small. Knowing this, we deduce (12) at once from (9) and from (8), which holds as an equality whenever $\left|x-\lambda_{n}\right|$ is bounded away from zero. Specifically, let $\epsilon>0$ be given, and choose $\delta_{1}$ so small that $\int_{a / x}^{\delta}$ and $\int_{1-\delta}^{1-b / x}$ in (9) are less than $\epsilon / 2$ whenever $\delta<\delta_{1}$. Then the assumption $\log ^{+}|F(x)|=o(x)$ combines with the equality (8) to give

$$
\lim _{x \rightarrow \infty} \int_{\delta}^{1-\delta} p(v, x) d v /\left(1-v^{2}\right)<\epsilon
$$

whenever $\delta<\delta_{1}$ and $\left|x-\lambda_{n}\right|$ is bounded away from zero; say $\left|x-\lambda_{n}\right|>c / 3$. If the result (21) held for all $x$ we should have established (12).

Suppose we alter $x$ by an amount $c$ or less. The change in $p(v, x)$ is at most $2 c / x$, since $\lambda_{n+1}-\lambda_{n}>c$; and this change does not affect the validity of (21). Since every value of $x$ is within a distance $c$ of a value $x$ satisfying $\left|x-\lambda_{n}\right|>c / 3$, (21) holds for all $x$.

The proof of Theorem VI is complete, then, as soon as we show that $\int_{1-\delta}^{1-b / x}$ in (9) is small for small $\delta$. For convenience we repeat the proof in [3], taking this opportunity to correct a numerical error. Divide the interval $(1-\delta, 1)$ into $[4 \delta x / c]$ subintervals each of length $c / 4 x$ (one at the end may be shorter). Now, $p(x, v)$ changes by at most $2 /\{x(1-\delta)-1\}$ when we proceed from one interval to the next, and this is $<6 / x$ for large $x$. So in the $k$ th interval from the right we have $p(v, x)<6 k / x$, since $p(1, x)=0$. The integral over the $k$ th interval does not exceed $6 / x$, by an easy calculation, so the whole integral does not exceed $24 \delta / c$, by summation.

The behavior of $\int_{1-\delta}^{1-b / x}$ is the crux in many of the questions with which we are concerned, since only here is the kernel $1 /\left(1-v^{2}\right)$ unbounded. It seems worth noting that simple changes of variable yield

$$
\int_{1-\delta}^{1-b / x} p(v, x) d v /\left(1-v^{2}\right)=\int_{b}^{\delta x}[\Lambda(x-w)-\Lambda(x+w)] d w / 2 w x
$$

apart from terms $O(\delta), O(1 / x)$. In this form the role of the condition $\lambda_{n+1}$ $-\lambda_{n}>c$ is transparent; it yields $\Lambda(x+w)-\Lambda(x-w)<2 w / c$ so that the integral $\left|\int_{a}^{\delta x}\right|$ does not exceed $\delta / c$, by inspection.

The complex plane. We wish to investigate further the distinction between the conditions $\log ^{+}|F(x)|=o(x)$ and $\lim \Lambda(u) / u=D$. Although precise description is difficult when attention is confined to the real axis, a discussion is easily given in terms of $F(z)$.

THEOREM VII. The following conditions are equivalent:

(a) $\lim \sup \log |F(x)| / x \leqq 0$ and $\lim \sup \Lambda(u) / u=D<\infty$.

(b) $\lim \sup \log \left|F\left(r e^{i \theta}\right)\right| / r=\pi D^{\prime}|\sin \theta|$ for some $D^{\prime}<\infty$ and all $\theta$. 
The discussion leading up to (5) gives the well known result [2]

$$
y^{-1} \log |F(i y)|=\int_{0}^{\infty} \frac{\Lambda(u)}{u} \frac{2 y}{u^{2}+y^{2}} d u
$$

so that (a) implies, again as in [2],

$$
\lim \sup \log |F(i y)| / y=\pi D^{\prime} \leqq \pi D
$$

(cf. Equation (32)). The Phragmén-Lindelöf function $h(\theta)$ for $F(z)$ therefore satisfies

$$
h(\theta) \leqq H(\theta)=\pi D^{\prime} \sin |\theta|
$$

when we note $\log ^{+}|F(x)|=o(x)$ by (a). Now, $h(\theta)$ is symmetric about the real axis, since $F(x)$ is real. If $h\left(\theta_{1}\right)<H\left(\theta_{1}\right)$ for some $\theta_{1}$ between 0 and $\pi / 2$, then $h\left(-\theta_{1}\right) \geqq H\left(-\theta_{1}\right)$ by [5]. This is impossible since $h\left(-\theta_{1}\right)=h\left(\theta_{1}\right)$, and hence (a) gives $(b)$.

That (b) implies (a) is trivial, since (b) ensures that $F(z)$ is of exponential type; and $\lim \sup \Lambda(u) / u<\infty$ for all such functions. The following considerations enable us to estimate $D$ in terms of $D^{\prime}$. If (b) is given, then [5] $\log \left|F\left(r e^{i \theta}\right)\right| / r<H(\theta)+\epsilon$ uniformly for $r>r_{0}(\epsilon)$. Hence Jensen's theorem

$$
\frac{1}{r} \int_{0}^{r} \frac{\Lambda(u)}{u} d u=\frac{1}{4 \pi r} \int_{0}^{2 \pi} \log \left|F\left(r e^{i \theta}\right)\right| d \theta
$$

gives $\lim \sup r^{-1} \int_{0}^{r}(\Lambda(u) / u) d u \leqq D^{\prime}$. This shows easily that $\lim \sup \Lambda(u) / u$ is finite; a detailed discussion is given later (lemma for Theorem XI).

In the presence of a mild restriction on $\Lambda(u)$, the condition $\log |F(x)|$ $\leqq o(x)$ is equivalent to a certain type of regular growth in the complex plane. Such is the content of Theorem VII. It will be seen, now, that $\lim \Lambda(u) / u=D$ is equivalent to a stronger regularity condition; and thus the two hypotheses, $\log |F(x)| \leqq o(x)$ and $\Lambda(u) \sim D u$, can be readily compared.

TheOREM VIII (PFLUGer). The following conditions are equivalent:

(a) $\lim \Lambda(u) / u=D$.

(b) Given any $\epsilon>0$ there is a constant $C=C(\epsilon)$ such that, for $\pi-\epsilon \geqq|\theta| \geqq \epsilon$, we have

$$
\exp (\pi D r|\sin \theta|-\epsilon r) \leqq\left|F\left(r e^{i \theta}\right)\right| \leqq \exp (\pi D r|\sin \theta|+\epsilon r)
$$

whenever $r>C$.

The condition (b) is almost enough to ensure that the $\lambda$ 's are real; the corresponding statement for complex $\lambda$ 's is the following:

Theorem IX (VALIRon-TitchmaRsh). Let $F(z)=\prod\left(1-z^{2} / \lambda_{n}^{2}\right)$ where $\lambda_{n}$ are any complex numbers such that $\sum\left|\lambda_{n}\right|^{-2}<\infty$. Then $\lim n / \lambda_{n}=D$, a real number, if and only if VIII(b) holds. 
Theorem VIII is essentially Theorem 3 of [7], and Theorem IX is essentially the Valiron-Titchmarsh theorem for entire functions of order $1 / 2$ (cf. $[9 ; 10 ; 13])$. Our procedure differs, however, from that used heretofore. We prove Theorem IX, which contains Theorem VIII; the method illustrates how some of the other results might be extended to complex $\lambda$ 's. Suppose first that VIII(b) holds. In the region $\pi-\epsilon \geqq|\theta| \geqq \epsilon$, there can be at most a finite number of zeros. Otherwise there would be a sequence $r_{n} \exp \left(i \theta_{n}\right)$ with $r_{n} \rightarrow \infty$, and the left-hand inequality of VIII(b) would be violated. It suffices, then, to prove $\lim \Lambda(u) / u=D$; the condition $\lim n / \lambda_{n}=D$ follows from this.

By Jensen's theorem (24) we have

$$
4 \pi \int_{0}^{r} \Lambda(u) d u / u=\left\{\int_{E}+\int_{E^{\prime}}\right\} \log \left|F\left(r e^{i \theta}\right)\right| d \theta
$$

where $E$ is the set $\pi-\epsilon \geqq|\theta| \geqq \epsilon$ and $E^{\prime}$ its complement. The fact that the $\lambda_{n}$ 's cluster about the real axis ensures that $|F(x)| \ll|F(i x)|$ for large $x$, so that (by the Phragmén-Lindelöf theorem, for example) $\int_{E^{\prime}}$ in (25) is $O(\epsilon r)$ as $r \rightarrow \infty$. Computing $\int_{E}$ by use of VIII(b), and noting that $\epsilon$ is arbitrary, we find $\lim r^{-1} \int_{0}^{r} \Lambda(u) d u / u=D$. Hence $\lim \Lambda(u) / u=D$, as was to be shown (cf. lemma for Theorem XI).

Suppose, now, that $\lim n / \lambda_{n}=D$, where $D$ is real. To deduce VIII(b), write $\lambda_{n}=r_{n} \exp \left(i \theta_{n}\right)$, with

$$
r_{n} \sim n / D, \quad \theta_{n} \rightarrow 0, \quad r_{n+1} \geqq r_{n} .
$$

Changing each $r_{n}$ by an arbitrarily small amount (insignificant for our estimates) enables us to assume $r_{n+1}>r_{n}$, which we do. In that case $\theta_{n}=g\left(r_{n}\right)$ for a function $g$, which is defined at other values of $r$ by $g(r)=\theta_{n}, r_{n} \leqq r$ $<r_{n+1}$. In this notation

$$
\log \left|F\left(r e^{i \theta}\right)\right|^{2}=\int_{0}^{\infty} \log \left|1-\frac{2 r^{2}}{u^{2}} \cos 2[\theta-g(u)]+\frac{r^{4}}{u^{4}}\right| d \Lambda(u)
$$

where $\Lambda(u)$ is the number of $r_{n}$ 's less than $u$. Given $\delta>0$ we have $|g(u)|<\delta$ for large $u$, since $g(u) \rightarrow 0$. Now, changing $\theta_{n}$ to zero in a finite number of factors enables us to assume $g(u)<\delta$ for all $u$, not merely for all large $u$. Since

$$
\lim _{r \rightarrow \infty}\left|1-z^{2} / \lambda^{2}\right| /\left|1-z^{2} / \lambda^{\prime 2}\right|=\left|\lambda^{\prime} / \lambda\right|^{2} \quad \text { for any } \lambda, \lambda^{\prime},
$$

this change in $F(z)$ does not affect the estimates with which we are concerned.

Let $\pi-\epsilon \geqq|\theta| \geqq \epsilon$, and choose $\delta<\epsilon / 2$. Then the absolute value in (26) is bounded away from zero; indeed, its minimum is $\sin ^{2}(\epsilon-\delta) \geqq \sin ^{2} \epsilon / 2$. Moreover, at each $u$ this absolute value is a monotonic function of the value of $g(u)$, provided $|\theta \pm \pi / 2|>\delta$. The expression (26) therefore lies between its value for $g(u) \equiv \delta$ and its value $g(u) \equiv-\delta$ in this case. If $|\theta-\pi / 2|<\delta$, the 
expression lies between its value for $g-\theta=0$ and for $g-\theta=2 \delta$. Writing $\pm \delta$ for $g(u)$ and integrating by parts gives

$$
4 \int_{0}^{\infty} \frac{\Lambda(u)}{u} \frac{r^{2} u^{2} \cos (\theta \pm \delta)-r^{4}}{u^{4}-2 r^{2} u^{2} \cos 2(\theta \pm \delta)+r^{4}} d u
$$

for the first case; discussion of the second is similar. Letting $u=r v$ gives

$$
4 r \int_{0}^{\infty} \frac{\Lambda(r v)}{r v} \frac{v^{2} \cos 2(\theta \pm \delta)-1}{v^{4}-2 v^{2} \cos 2(\theta \pm \delta)+1} d v .
$$

We write the integral as $\int_{0}^{\delta}+\int_{\delta}^{\infty}$. Since the integrand is bounded the first is $O(\delta)$, uniformly in $\theta$ and $r$. For the same reason the second tends uniformly, as $r \rightarrow \infty$, to the value it would have for $r=\infty$; that is, to the value with $\Lambda(r v) /(r v)$ replaced by $D$. Finally, this latter integral differs, uniformly, by $O(\delta)$ from its value with $\delta=0$. Choosing $\epsilon$ first, then a sufficiently small $\delta$, and finally $r$, we obtain the theorem. (Evaluation of the integral is avoided since the corresponding $F(z)$ is, essentially, $\cos (\pi D z)$ ).

Theorem IX has the following corollary:

Corollary. Let $F(z)=\prod\left(1-z^{2} / \lambda_{n}^{2}\right), \quad \lim n / \lambda_{n}=D$, and $G(z)$ $=\prod\left(1-z^{2} / \mu_{n}^{2}\right), \lim n / \mu_{n}=D^{\prime}$, with $D, D^{\prime}$ real and $D^{\prime} \neq D$. Then the roots $\pm z_{n}$ of the equation $F(z)=G(z)$ satisfy $\lim n / z_{n}=\max \left(D, D^{\prime}\right)$.

For proof, observe that VIII(b) holds for $F(z)$ and, with $D^{\prime}$ instead of $D$, for $G(z)$. Hence VIII (b) holds for $F(z)-G(z)$, with $D$ replaced by max $\left(D, D^{\prime}\right)$. The condition $D \neq D^{\prime}$ is of course essential. If $D^{\prime}=D$ then $\lim n / z_{n}$ need not exist; and when it does exist, it can be any number between 0 and $D$.

Upper limits, lower limits, and inequalities. We define

$$
\begin{array}{ll}
A=\lim \sup \log |F(i y)| / y, & a=\lim \text { inf, } \\
B=\lim \sup \frac{1}{r} \int_{0}^{r} \frac{\Lambda(u)}{u} d u, & b=\lim \text { inf, } \\
C=\lim \sup \frac{1}{4 \pi r} \int_{0}^{2 \pi} \log \left|F\left(r e^{i \theta}\right)\right| d \theta, & c=\lim \inf , \\
D=\lim \sup \Lambda(u) / u, & d=\lim \text { inf, }
\end{array}
$$

where each variable approaches infinity. It is pointed out in [2] that $A \leqq \pi D$, and that $D$ is finite if $A$ is; the latter is a simple consequence of Jensen's theorem. These results suggest the following:

Theorem X. Let $\tau=2(s-1)^{1 / 2} / s$, where $s$ is the positive root of $s \log s$ $=2(s-1)$. Then $\pi D \geqq A \geqq \tau D$, and this is best possible. Moreover, if $A=\tau D$ then $d=0$.

We have $s \cong 2.9213, \tau \cong 0.8047$. To prove that $A \geqq \tau D$, let $\Lambda(u) / u=D^{\prime} \geqq D$ 
$-\epsilon$ at $u=p$. That this holds for arbitrarily large $p$ and arbitrarily small $\epsilon$ follows from the definition of $D$. Let $L(u)$ be defined as 0 for $0<u<p$, and as $D^{\prime} p$ for $p \leqq u<\infty$. The non-negative increasing character of $\Lambda(u)$ ensures that $\Lambda(u) \geqq L(u)$, so that the integral (23) dominates the corresponding expression with $L(u)$. We have

$$
\int_{0}^{\infty} \frac{L(u)}{u} \frac{2 y}{u^{2}+y^{2}} d u=\left(D^{\prime} / t\right) \log \left(1+t^{2}\right), \quad t=y / p .
$$

Differentiation shows that (29) is maximum when

$$
\left(1+t^{2}\right) \log \left(1+t^{2}\right)=2 t^{2}
$$

and that the maximum is then $2 t /\left(1+t^{2}\right)$. Writing $s=1+t^{2}$ gives the righthand inequality in Theorem $\mathrm{X}$. That the result is best possible follows, as usual, by modifying $L(u)$ so that it returns infinitely of ten to $D u$.

If $d>0$, we define $L(u)$ as $d^{\prime} u$ for $0<u<p$, as $p D^{\prime}$ for $p<u<q$, and as $d^{\prime} u$ for $q \leqq u<\infty$. Here $d^{\prime} \geqq d-\epsilon$, and $p D^{\prime}=q d^{\prime}$. The integral (29) is easily computed; it is

$$
d^{\prime}\left\{2 \tan ^{-1}(1 / t)+(m / t) \log m^{2} \frac{1+t^{2}}{m^{2}+t^{2}}+\pi-2 \tan ^{-1}(m / t)\right\}
$$

where $m=D^{\prime} / d^{\prime}, t=y / p$. Equation (23) does not quite dominate (31), since we may have $\Lambda(u)<L(u)$ for $u$ small. Writing (23) as

$$
\left\{\int_{0}^{\delta}+\int_{\delta}^{\infty}\right\} \frac{\Lambda(y v)}{y v} \frac{2 v d v}{1+v^{2}}
$$

shows, however, that $\Lambda(u)$ for small $u$ has no influence on the estimates with which we are concerned. Hence (31) is dominated when $p$ and $y$ are large. Since $d^{\prime} \rightarrow d, D^{\prime} \rightarrow d$ as $p=p_{i} \rightarrow \infty$, the upper limit of $y^{-1} \log \left|F^{\prime}(i y)\right|$ dominates (31), with $d^{\prime}=d, D^{\prime}=D$.

It follows that $A>\tau D$. For, (31) as it stands exceeds (31) with $d^{\prime}=0$ (this is plain from the derivation, though direct verification is tedious). Hence the maximum of (31) exceeds the maximum of (29); and if $A=\tau D$, we must therefore have $d=0$.

The theorem can be extended to the case in which $d$ as well as $D$ is prescribed, although the resulting formulation is complicated and inelegant. By simply putting $t=1$ in (31), however, we find the right-hand inequality, which is not optimum, in

$$
\pi D \geqq A \geqq 3 \pi d / 2+D \log \frac{2 D^{2}}{d^{2}+D^{2}}-2 d \tan ^{-1} \frac{D}{d} .
$$

Similarly, by considering a function $L(u)$ defined as $D u$ for $0<u<p$, as $D p$ for $0 \leqq u<q$, and as $D u$ for $q<u<\infty$, we find 


$$
\pi d \leqq a \leqq 3 \pi D / 2+d \log \frac{d^{2}+D^{2}}{2 d^{2}}-2 D \tan ^{-1} \frac{D}{d}
$$

when we put $t=1 / m$ in the expression corresponding to (31).

THEOREM XI. In the notation (28) we have

$$
\begin{gathered}
d \leqq c \leqq C \leqq D, \\
1+\log (C / D) \geqq c / D, \quad 1+\log (C / d) \geqq c / d .
\end{gathered}
$$

Moreover these inequalities are best possible. Given any $C, c, D, d$ satisfying them, there is a corresponding $F(z)$.

We shall establish this result for all entire functions (defining $2 \Lambda(u)$ = number of $\left.\left|\lambda_{n}\right| \leqq u\right)$, not only for the $F(z)$ in (2). Theorem XI gives the following Tauberian theorem:

Corollary 1. If $\lim (1 / 4 \pi r) \int_{0}^{2 \pi} \log \left|F\left(r e^{i \theta}\right)\right| d \theta=D$, then $\lim \Lambda(u) / u=D$; and hence, when $F(z)$ is given by (2), we have VIII(b).

Also it enables us to make the result VII more precise:

COROLLARY 2. In Theorem VII we have $e D^{\prime} \geqq D \geqq D^{\prime}$ (where $e$ is the base of natural logarithms).

Theorem $\mathrm{X}$ yields only the weaker inequality $\pi D^{\prime} / \tau \geqq D \geqq D^{\prime}$. Since Jensen's theorem gives $C=B, c=b$, it suffices to establish the following lemma:

Lemma. In the notation (28) we have $d \leqq b \leqq B \leqq D, 1+\log (B / D) \geqq b / D$, $1+\log (B / d) \geqq b / d$, and these are best possible. If $b=B$, then $D=d$.

The second part of the lemma follows from the first when we note that $1+\log x<x$ for $x>0, x \neq 1$. Thus, $f(x)=1+\log x-x$ satisfies $f^{\prime}(x)=\left(1-x^{2}\right) / x$, so that $x=1$ gives the maximum; but $f(1)=0$. (The second statement also follows from a general Tauberian theorem of Wiener, or from a similar theorem of Hardy for sums.)

To prove the first part of the lemma, let $\Lambda(u) / u=D^{\prime}>D-\epsilon$ at $u=p$. This happens for a sequence $p=p_{i} \rightarrow \infty$ and $\epsilon=\epsilon_{i} \rightarrow 0$. In order not to confuse an essentially simple proof, we drop the primes, writing $B, b, D, d$ for quantities arbitrarily close (when $p$ is large) to $B, b, D, d$. Define $L(u)$ as $b u$ for $0<u<p$ and as $D p$ for $p \leqq u \leqq q$, where $D p=d q$. We have

$$
\frac{1}{r} \int_{0}^{r} \frac{L(u)}{u} d u=x b-D x \log x, \quad x=p / r .
$$

The maximum occurs at $\log x=(b-D) / D$, which is interior to the interval $p<r<q$. For, with $m=b / D$ we have $x=e^{m-1} \leqq 1$ since $m<1$. On the other hand if $f(m)=e^{m-1}-m$ we have $f(1)=0, f^{\prime}(m)=e^{m-1}-1 \leqq 0$; so $e^{m-1}<m$. 
The maximum value is $D e^{(b / D-1)}$.

It will be seen, now, that the maximum for $\Lambda(u)$ is just as large. We have

$$
\frac{1}{r} \int_{0}^{r} \frac{\Lambda(u)}{u} d u=\frac{p}{r} \frac{1}{p} \int_{0}^{p}+\frac{1}{r} \int_{p}^{r} .
$$

By the definition of $b$,

$$
\frac{1}{p} \int_{0}^{p} \frac{\Lambda(u)}{u} d u \geqq b,
$$

and since $\Lambda(u)$ is nondecreasing we have $\Lambda(u) \geqq L(u)$ for $u>p$. Thus,

$$
\frac{1}{r} \int_{0}^{r} \frac{\Lambda(u)}{u} d u \geqq \frac{1}{r} \int_{p}^{r} \frac{L(u)}{u} d u .
$$

Combining these inequalities gives

$$
\frac{1}{r} \int_{0}^{r} \frac{\Lambda(u)}{u} d u \geqq x b-D x \log x
$$

as was required (see (35)). Comparing maxima yields

$$
B \geqq D e^{(b / D-1)}
$$

which is the first nontrivial inequality of the lemma.

Suppose next that $\Lambda(u) / u=d$ at $u=s$ (more accurately, $\Lambda(u) / u=d^{\prime}<d+\epsilon$ for a sequence $\left.u=s_{i} \rightarrow \infty, \epsilon=\epsilon_{i} \rightarrow 0\right)$. Define $L(u)$ as $d s$ for $p<u<s$, where $p B=d s$, and as $B u$ for $0<u \leqq p$. If $p<r<s$ we have

$$
\frac{1}{r} \int_{0}^{r} \frac{L(u)}{u} d u=B(x-x \log x), \quad x=p / r,
$$

which decreases steadily to a minimum value $d(1-\log [d / B])$ at $r=s$. By definition of $B$, for large $p$ we have

$$
\frac{1}{p} \int_{0}^{p} \frac{\Lambda(u)}{u} d u \leqq B
$$

and since $\Lambda(u)$ is nondecreasing, $\Lambda(u) \leqq L(u)$ for $p<u<s$. It follows as before that

$$
\frac{1}{r} \int_{0}^{r} \frac{\Lambda(u)}{u} d u \leqq \frac{1}{r} \int_{0}^{r} \frac{L(u)}{u} d u
$$

whence $b \leqq d(1-\log (d / B))$.

To show that the result is optimum, it suffices to give a $\Lambda(u)$ for which both the relations become equalities. Define $\Lambda(u)=b u$ up to $u=p_{1}$, then $\Lambda(u)=D p_{1}$ until $\Lambda(u)=B u$, then $\Lambda(u)=B u$ up to a large value $p_{2}$, then 
$\Lambda(u)=B p_{2}$ until $\Lambda(u)=d u$, then $\Lambda(u)=b u$ to $p_{3}$, and so on. Since the behavior of $\Lambda(u)$ for small $u$ has no influence on our estimates, the sequence $p_{i}$ can be so chosen that both equalities are obtained. Certainly $p_{n} / p_{n-1} \rightarrow \infty$ suffices.

Other inequalities and a Tauberian theorem. The following relates to certain functions having, on the average, only a few zeros:

THEOREM XII. When the right-hand side is finite,

$$
\begin{gathered}
\int_{0}^{\infty}\left|\frac{\log |F(i y)|}{y}\right|^{2} \frac{d y}{y}<\pi^{2} \int_{0}^{\infty}\left[\frac{\Lambda(u)}{u}\right]^{2} \frac{d u}{u}, \\
\int_{0}^{\infty}\left|\frac{F^{\prime}(i y)}{F(i y)}\right|^{2} \frac{d y}{y}<\pi^{2} \int_{0}^{\infty}\left[\frac{\Lambda(u)}{u}\right]^{2} \frac{d u}{u} .
\end{gathered}
$$

It is easy to see that

$$
\int_{0}^{\infty}\left|\frac{\log |F(i y)|}{y}\right|^{2} \frac{d y}{y}=2 \int_{0}^{\infty} \int_{0}^{\infty} \frac{\Lambda(t)}{t} \frac{\Lambda(v)}{v} \frac{\log t^{2}-\log v^{2}}{t^{2}-v^{2}} d v d t,
$$

with a similar expression for the other integral, but we have not been able to carry out a proof along these lines. To establish Theorem XII, let $y=e^{s}$, $u=e^{-t}$ in

$$
y^{-1} \log |F(i y)|=\int_{0}^{\infty} \frac{\Lambda(u y)}{u y} \frac{2 d u}{1+u^{2}}
$$

to find

$$
\phi(s)=e^{-s} \log \left|F\left(i e^{s}\right)\right|=f^{*}\left((2 \pi)^{1 / 2} \operatorname{sech} t\right)
$$

where $f(u)=\Lambda\left(e^{u}\right) / e^{u}$ and the ${ }^{*}$ denotes convolution. We have

$$
\int_{-\infty}^{\infty}|f(u)|^{2} d u=\int_{0}^{\infty}\left[\frac{\Lambda(t)}{t}\right]^{2} \frac{d t}{t}=I .
$$

If $T$ denotes Fourier transformation in the mean square sense, therefore,

$$
T \phi=(T f)\left(T(2 \pi)^{1 / 2} \operatorname{sech} t\right)=(T f)(\pi \operatorname{sech} \pi x / 2) .
$$

By the Plancherel theorem

$$
\begin{aligned}
\int_{-\infty}^{\infty}|\phi|^{2} & =|T \phi|^{2}=\int|T f|^{2} \pi^{2} \operatorname{sech}^{2}(\pi x / 2) d x \\
& <\pi^{2} \int|T f|^{2}=\pi^{2} \int|f|^{2}=\pi^{2} I
\end{aligned}
$$

which is the first inequality. For the second, differentiating (23) yields 


$$
\begin{aligned}
i \frac{F^{\prime}(i y)}{F(i y)} & =\int_{0}^{\infty} \frac{\Lambda(t)}{t} \frac{4 y t^{2}}{\left(t^{2}+y^{2}\right)^{2}} d t=\int_{0}^{\infty} \frac{\Lambda(u y)}{u y} \frac{4 u^{2} d u}{\left(1+u^{2}\right)^{2}} \\
& =f^{*}\left((2 \pi)^{1 / 2} e^{-t} \operatorname{sech}^{2} t\right)
\end{aligned}
$$

as before. The maximum of $T\left((2 \pi)^{1 / 2} e^{-t} \operatorname{sech}^{2} t\right)$ occurs at $x=0$, and equals

$$
4 \int_{-\infty}^{\infty} \frac{e^{-t}}{\left(e^{t}+e^{-t}\right)^{2}} d t=4 \int_{0}^{\infty} \frac{x^{2} d x}{\left(1+x^{2}\right)^{2}}=\pi \text {. }
$$

The remainder of the proof follows (39). It is probable that a sharper statement could be given by contour integration, though we have not done so. The following is an immediate consequence of Theorem XII:

COROllary. There is a function with $\lim \sup \log |F(i y)| / y=D$ and with

$$
\int_{0}^{\infty}\left|\frac{\log |F(i y)|}{y}\right|^{2} \frac{d y}{y}<(\pi D)^{2} / 2 \text {. }
$$

In connection with $\mathrm{XII}$, the following Tauberian theorem is of interest:

Theorem XIII. $\lim i F^{\prime}(i y) / F(i y)=\pi D$ if and only if $\lim \Lambda(u) / u=D$.

The proof is practically contained in a discussion of Paley and Wiener [2], where they establish other theorems of the same character. Differentiation of

$$
\log |F(i y)|=\int_{0}^{\infty} \log \left(1+y^{2} / v^{2}\right) d \Lambda(v)
$$

gives

$$
(i / \pi) F^{\prime}(i y) / F(i y)=y^{-1} \int_{0}^{\infty} K(v / y) d \Lambda(v)
$$

where $K(u)=(2 / \pi)\left(1+u^{2}\right)^{-1} . K(u)$ is nonincreasing, $\int_{0}^{\infty} K(u) d u=1$, and

$$
\int_{0}^{\infty} K(u) u^{i w} d u=\pi^{-1} \int_{-\infty}^{\infty} \operatorname{sech} x e^{-i w v} d x=\operatorname{sech} \pi x / 2 \neq 0 .
$$

Hence Theorem XIII is immediate, by the theorem of Wiener cited in [2].

Uniformly distributed zeros. If $|\Lambda(u)-D u| \leqq H$ for some constants $H$ and $D$, we shall say that the zeros are distributed uniformly. By estimates based on the gamma function, Paley and Wiener [2] have shown that $|F(x)|<C|x|^{4 H-1}$ where $C$ is a constant depending on $F(x), D$, and $H$. Using a different procedure we improve the exponent to $4 H-2$, show that the result is best possible (in a certain special sense), and estimate the constants. Related results are given in [11].

TheOREM XIV. If $|\Lambda(u)-D u| \leqq H$, with $H>1 / 2, D>0$, then $|F(x)|$ 
$<C_{1} x^{4 H-2}$ for some constant $C_{1}$ depending on $D, H$, and $\lambda$, the first zero of $F(x)$, but not otherwise on $F(x)$, or on $x$. If $x$ is given there exist a similar constant $C_{2}>0$ and a function $F(x)$ (depending on $x$ ) such that $|\Lambda(u)-D u| \leqq H$ and $|F(x)| C_{2} x^{4 H-2}$. If $D=0$ then $|F(x)| \leqq(x / \lambda)^{[H]}$, where $\lambda$ is the first zero of $F(x)$, and this is optimum. If $H=1 / 2$, then $|F(x)| \leqq 1$.
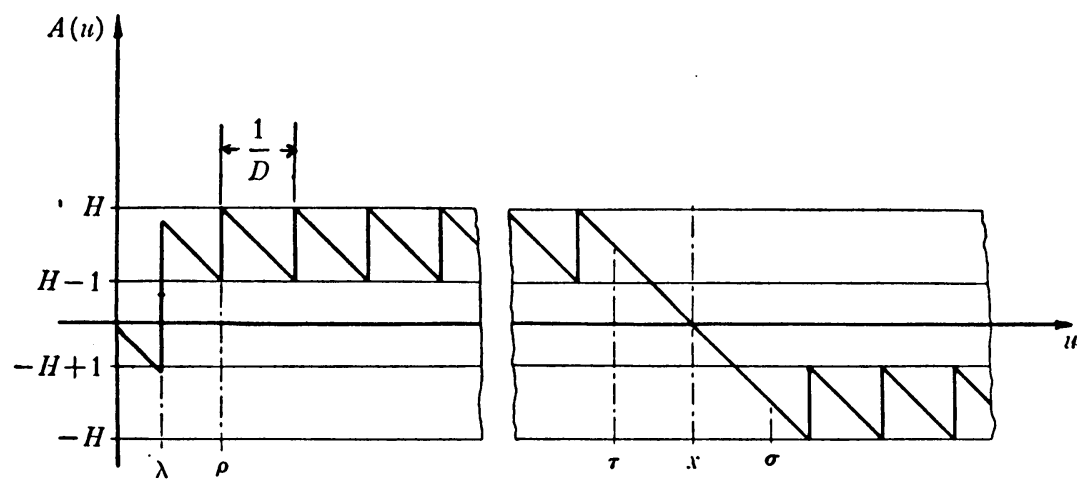

FIG. 1. A function $A(u)=\Lambda(u)-D u$ for which $|A(u)| \leqq H$ and $|F(x)|>C x^{4 H-2}$.

When $D=0$ then $F(x)$ is a polynomial, so that the result is trivial; and when $H=1 / 2$ we must have $F(x)=\cos \pi D x$. We confine our attention therefore to the case $H>1 / 2, D>0$. For large $x$ (that is, apart from $O(1 / x)$ ) one may choose

$$
\begin{aligned}
& C_{2}=\left\{\frac{e D}{(2 H-1) \lambda}\right\}^{2 H-1}\left(\frac{\lambda}{\rho}\right)\left(\frac{\rho}{e \lambda}\right)^{2 \rho D}, \quad e=2.718 \cdots, \\
& C_{1}=C_{2}\left(1+\frac{1}{D \rho}\right)^{2 H}\left(\frac{2 H+1}{2 H-1}\right)^{2 H}
\end{aligned}
$$

where $C_{2}$ in (42) is given by (41), and where

$$
D \rho=[H+D \lambda]+1-H,
$$

so that $\lambda<\rho \leqq \lambda+1 / D$. Of course, $\lambda \leqq H / D$ is assumed.

The proof is elementary but somewhat long because one must make essential use of the fact that $\Lambda(u)$ is an integer. (The integral character of $\Lambda(u)$ has played no role in our considerations heretofore.) To begin with, Equation (7) yields

$$
\log |F(x)|=\left\{\int_{\lambda}^{x}+\int_{x}^{\infty}\right\} A(u) K(x, u) d u-2 \lambda D+O(1 / x)
$$

where $K(x, u)=2 x^{2} /\left[u\left(x^{2}-u^{2}\right)\right]$, and where the integral near $x$ is a Cauchy principal value. In our applications $x \neq \lambda_{n}$. The $O(1 / x)$ term is uniform for the 
whole class of $F(x)$ having a given $\lambda$ and $\max \Lambda(u) / u$. We must estimate the integral (44), when $|A(u)| \leqq H$.

The function $A(u)=\Lambda(u)-D u$ is composed of vertical straight segments having integral lengths, at the zeros of $F(x)$, and of straight segments having slope $-D$ between them. For $u<x$ we have $K(x, u)>0$ and for $u>x$ we have $K(x, u)<0$. Hence the function $A(u)$ which maximizes $F(x)$ at a particular $x$ is as large as possible for $u<x$, and as small as possible for $u>x$. It has, in fact, the general character illustrated in Fig. 1. (Although we have not proved that this is the maximizing function, it does furnish the example $|F(x)|$ $>C_{2} x^{4 H-2}$, as we shall see later.) Evidently, $A(u)$ behaves like $H-1 / 2$ for $u<x$, and like $-H+1 / 2$ for $u>x$. This observation suggests Theorem XIV. A proof can be based on the following lemma, which is needed also for estimating $C_{1}$ and $C_{2}$ :

LEMMA 1. Let $K(u)$ be non-negative. If $K(u)$ is increasing then

$$
\int_{p}^{p+1 / D}(A-1 / 2) K(u) d u \geqq \int_{p-1 / D}^{p} A(u) K(u) d u
$$

for each $p$; and if $K(u)$ is decreasing then

$$
\int_{p-1 / D}^{p}(A-1 / 2) K(u) d u \geqq \int_{p}^{p+1 / D} A(u) K(u) d u .
$$

Let $A(u)$ attain its maximum in $[p-1 / D, p]$ at $u=q$. At $u>q$ of the curve, $A(u)$ follows a straight line of slope $-D$ (otherwise it would reach a higher maximum) and at $q, A(u)$ drops at least by unity for the same reason. Hence $A(u)$ is majorized by the function which is equal to $H$ at $q+$, to $H-1$ at $q$ and to a linear function of slope $-D$ elsewhere. For this $A(u)$ we have

$$
\begin{aligned}
\int_{p-1 / D}^{p} A(u) K(u) d u & \leqq K(p) \int_{p-1 / D}^{p} A(u) d u=K(p) \int_{p-1 / D}^{p}(A-1 / 2) d u \\
& \leqq \int_{p}^{p+1 / D} K(u)(A-1 / 2) d u .
\end{aligned}
$$

The other part of the lemma is proved in the same way.

To prove the theorem, we observe that $K(x, u)$ is decreasing for $u<x / 3^{1 / 2}$, increasing for $u>x / 3^{1 / 2}, u \neq x$. By (46) of the lemma, therefore,

$$
\int_{p-1 / D}^{p}(H-1 / 2) K \geqq \int_{p}^{p+1 / D} A K, \quad p+1 / D<x / 3^{1 / 2},
$$

so that, taking $p=\lambda+1 / D, \lambda+2 / D, \cdots$ and adding,

$$
\int_{\lambda}^{q}(H-1 / 2) K \geqq \int_{\lambda+1 / D}^{q+1 / D} A K
$$


where $q=\lambda+m / D, m$ an integer; $q$ is so chosen that $q+1 / D<x / 3^{1 / 2}, \mid q$ $-x / 3^{1 / 2} \mid<2 / D$. The precise value is immaterial; the terms for $u \cong x / 3^{1 / 2}$ are $O(1 / x)$. For the interval $(\lambda, \lambda+1 / D)$ we have, of course,

$$
\int_{\lambda}^{\lambda+1 / D} H K \geqq \int_{\lambda}^{\lambda+1 / D} A K .
$$
find

Turning now to the interval $\left(x / 3^{1 / 2}, x-a\right)$ we use (42) of the lemma to

$$
\int_{s}^{x-a}(H-1 / 2) K \geqq \int_{s-1 / D}^{x-a-1 / D} A K
$$

where $s=x-a-n / D$ for some integer $n$, and $s$ is close to $x / 3^{1 / 2}$. We have also

$$
\int_{x-a-1 / D}^{x-a} H K \geqq \int_{x-a-1 / D}^{x-a} A K .
$$

The same procedure gives, $K$ being now negative,

$$
\begin{aligned}
-\int_{x+a}^{\infty}(H-1 / 2) K & \geqq \int_{x+a+1 / D}^{\infty} A K, \\
-\int_{x+a}^{x+a+1 / D} H K & \geqq \int_{x+a}^{x+a+1 / D} A K .
\end{aligned}
$$

By addition of (47)-(52) we obtain the first assertion of the theorem, after evaluating the elementary integrals for $a \rightarrow 0, x \rightarrow \infty$.

For the second part of the theorem, we use the lemma to estimate $F(x)$ corresponding to $A(u)$ in Fig. 1. The first zero, $\rho$, after the multiple zeros at $\lambda$, is given by (43). Making no attempt to determine the best constant, one can use the estimates

$$
\int_{\rho}^{\rho+1 / D} A K>\int_{\rho+1 / D}^{\rho+2 / D}(H-1 / 2) K, \quad \rho<u<x / 3^{1 / 2},
$$

and so on; the proof is very similar to that just given.

This procedure, and that described for showing $|F(x)| \leqq C x^{4 H-2}$, yield values for $C_{1}$ and $C_{2}$ which are less precise than those indicated above. To obtain estimates as sharp as (41), (42) the following lemmas are required:

Lemma 2. Let $A(u)$ be a linear function joining the points $(p, H)$ and $(p+1 / D, H-1)$. If $K(u)$ is a decreasing function, then

$$
\int_{p}^{p+1 / D} A K>\int_{p}^{p+1 / D}(H-1 / 2) K .
$$

Lemma 3. Let $A(u)$ in Lemma 2 be defined to have period $1 / D$. If $K(u)$ is 
increasing, then $\int_{p}^{p+1 / D} A(u+1 / 2 D) K>\int_{p}^{p+1 / D}(H-1 / 2) K$.

We omit the trivial proofs. By Lemma 2,

$$
\int_{\rho}^{q} A K>\int_{\rho}^{q}(H-1 / 2) K
$$

where $q$ is close to $x / 3^{1 / 2}$. Similarly, Lemma 3 gives

$$
\int_{s}^{\tau} A K>\int_{s}^{\tau}(H-1 / 2) K, \quad \int_{\sigma}^{\infty} A K>-\int_{\sigma}^{\infty}(H-1 / 2) K
$$

where $s$ is also close to $x / 3^{1 / 2}$. (The integral from $q$ to $s$ is $O(1 / x)$, hence insignificant.) Here $\sigma$ and $\tau$ are the values of $u$ at which the lines $y= \pm(H-1 / 2)$ intersect the line $y=D(x-u)$ (see Fig. 1). Evaluating the integral from $\tau$ to $\sigma$ and from $\lambda$ to $\rho$ exactly, and using (54), (55) elsewhere, gives (41).

To obtain (42) one must show that the $F$ which maximizes $F(x)$ will have no zero in the neighborhood of $x$, so that $A(u)$ crosses the point $(x, 0)$ in the manner illustrated by Fig. 1.

Lemma 4. If $x$ is large, we have

$$
\int_{x-H / D}^{x+H / D} A K \leqq \int_{x-H / D}^{x+H / D} D(x-u) K
$$

Let the curve $y=A(u)$ cross the vertical line $u=x$ at some point $y_{0}$. Since $K$ is negative for $u>x$, the integral for $u>x$ is majorized by a line of slope $-D$ passing through $\left(x, y_{0}\right)$. (This is as small as $A(u)$ can be, in view of its geometric properties, if $A(x)=y_{0}$.) Similarly, since $K$ is positive for $u<x$, the integral is majorized by the same line of slope $-D$, extended backwards from $\left(x, y_{0}\right)$. The maximizing function therefore crosses $u=x$ as a line of slope $-D$ joining $y=H$ and $y=-H$. It remains to show that $y_{0}=0$ is the optimum choice.

For a value $c \geqq 2 H / D$ we evaluate $\int_{x-c}^{x+c} A K$ where $A(u)$ follows $y=H$ to the line of slope $-D$ discussed above, then follows this line to $y=-H$, and follows $y=-H$ to $u=x+c$. If the line crosses the $u$ axis at $u=a$, the integral is

$$
\begin{aligned}
-D^{-1} \int_{x-c}^{x+c} A K= & (b+a) \log (b+a)+(b-a) \log (b-a) \\
& +2 b(\log c+1)+O(1 / x),
\end{aligned}
$$

where $b=H / D$. The terms involving $a$ are found by differentiation to be least at $a=0$, so that the integral is then maximum.

To obtain (42), we note that the integral from $\lambda$ to $\rho$ cannot exceed the value given by Fig. 1, as is easily proved; and we have just seen that the same is true for the integral from $\sigma$ to $\tau$. Dominating the integral elsewhere by 
adjacent intervals as in the discussion (47)-(52) introduces three new terms in addition to those used in the derivation of (41), namely,

$$
\int_{\rho}^{\rho+1 / D} H K, \quad \int_{\sigma-1 / D}^{\sigma} H K, \quad-\int_{\tau}^{\tau+1 / D} H K .
$$

These yield the extra factor in (42).

It is probable that (41) is a better estimate of the true constant than (42), as we see from the derivation. For $u<x / 3^{1 / 2}$, the function in Fig. 1 actually majorizes the integral, as we shall now show.

With $K(u)>0$ decreasing, it suffices to prove that

$$
\int_{0}^{1 / D}(1-D u) K(u) d u>\int_{0}^{1 / D} A(u) K(u) D u .
$$

This will follow as in the proof of Lemma 1 if the left side exceeds

$$
\int_{0}^{a} D(a-u) K+\int_{a}^{1 / D}[1+D(a-u)] K(u), \quad 0<a<1 / D .
$$

It suffices, therefore, to show that

$$
\int_{0}^{a}(1-D a) K>\int_{a}^{1 / D} D a K
$$

or that $\int_{0}^{a} K>\int_{a}^{1 / D} D a K$. Since $K$ is decreasing, however, it is true that

$$
\frac{1}{a} \int_{0}^{a} K>\frac{1}{(1 / D)} \int_{0}^{1 / D} K, \quad a<1 / D .
$$

Whether the $A(u)$ in Fig. 1 also maximizes the integral for $u>x / 3^{1 / 2}$ is problematical.

The main theorem on uniform distribution. In the preceding discussion the function $F(x)$ was allowed to depend on $x$, the point at which our estimates were made. The results were concerned with the whole class of functions having a given $\lambda, D$, and $H$. If, instead, one and the same $F$ is considered for a sequence $x=x_{i} \rightarrow \infty$, the growth of $F(x)$ is given by the following theorem:

Theorem XV. When $|\Lambda(u)-D u| \leqq H$ with $D>0, H>1 / 2$, then $F(x)$ $=o\left(x^{4 H-2}\right)$. Moreover, let $c(x)$ be any function for which $\lim c(x)=0$. Then there exists a distribution $\Lambda(u)$ such that $|\Lambda(u)-D u| \leqq H$ and such that $F(x)$ $>c(x) x^{4 H-2}$ for an infinite sequence $x=x_{i} \rightarrow \infty$. which

Suppose the theorem is not true. Then there is a sequence $x=x_{i} \rightarrow \infty$ for

$$
\log |F(x)|>4 S \log x-C_{1}, \quad S=H-1 / 2,
$$


where $C_{i}$ represent constants independent of $x$ for large $x$. As in the proof of Theorem XIV, for a fixed $a>0$

$$
\int_{\lambda}^{x-a} A(u) K(x, u) d u<3 S \log x+C_{2} .
$$

Also

$$
\int_{2 x}^{\infty} A(u) K(x, u) d u=O(1)
$$

by a simple calculation. From (56)-(58) it follows that

$$
\int_{x+a}^{2 x} A(u) K(x, u) d u>S \log x-C_{3}
$$

when $x=x_{i} \rightarrow \infty$.

The idea of the proof is to show that $A(u)$ must be negative in an appreciable fraction of the interval $(x+a, 2 x)$. This behavior for $x_{0}, x_{1}, \cdots, x_{n-1}$ prevents the inequality (56) for $x_{n}$ when $n$ is large.

Lemma. Let $E$ denote the set in $(x+a, 2 x)$ where $A(u)<0$. If $x=x_{i}, a$ value where (56) holds, then $m(E)>\delta x$ for some nonzero constant $\delta$.

Since $K(x, u)$ in (59) is negative and increasing, the most favorable case (that is, the case permitting smallest measure) arises when $E$ extends from $x+a$ to $\alpha x$. Although $|A(u)| \leqq S+1 / 2$ is postulated, rather than $|A(u)| \leqq S$, the proof of Theorem XIV shows that $A(u)$ behaves in our calculations as if $|A(u)| \leqq S$. This observation is used repeatedly in the sequel. In the present discussion, we have

$$
\int_{x+a}^{2 x} A K \leqq \int_{x+a}^{\alpha x} A K \leqq \int_{x+a}^{\alpha x}(-S) K \sim S \log x-S \log \alpha^{2} /\left(\alpha^{2}-1\right) .
$$

In view of (59) we must have, when $x$ is large,

$$
S \log \alpha^{2} /\left(\alpha^{2}-1\right)<C
$$

so that $\alpha>1+\delta$ for some fixed positive $\delta$. This yields the lemma. As a consequence

$$
\int_{x+a}^{2 x} A(u) 2 d u / u \leqq 2 S \log (2-\delta)+O(1 / x)
$$

where $\delta$ is the $\delta$ of Lemma 1 . For, since $2 / u$ is positive and decreasing, the most favorable case arises when $E$ is the interval $(2 x-\delta x, 2 x)$. We have then

$$
\int_{x+a}^{2 x}(2 / u) A(u) d u \leqq \int_{x+a}^{2 x-\delta x}(2 / u) A(u) d u \leqq \int_{x+a}^{2 x-\delta x}(2 / u) S d u
$$


and this is $(60)$.

To prove the theorem, consider a sequence $1 \ll x_{0}<x_{1}<\cdots<x_{n}$ for which (56) holds. We may assume $x_{n} \gg x_{n-1}$, so that $|K(u, y)-2 / u|<1 / x_{n-1}^{2}$, whence

$$
\int_{x+a}^{2 x} K A=\int_{x+a}^{2 x}(2 / u) A+O(1 / x)
$$

for $y \geqq x_{n}, x \leqq x_{n-1}$. This simplification (which is not necessary) is possible because $\lim K(u, y)=2 / u$ as $y \rightarrow \infty$. If $A(u)$ were so defined as to maximize $F\left(x_{n}\right)$ we should have

$$
\int_{x+a}^{2 x} A(u) K(u, x) d u=2 S \log 2+O(1 / x)
$$

for $x=x_{1}, \cdots, x_{n}$. Even in this case, however,

$$
\log \left|F\left(x_{n}\right)\right|<4 S \log x_{n}+C_{5}
$$

by Theorem XIV. The function $A(u)$ which we actually have satisfies (60) rather than (61). For each $x_{i}$, then, there is a default at least equal to $2 S \log 2 /(2-\delta)$. The total default is $n$ times this, so that

$$
\log \left|F\left(x_{n+1}\right)\right|<4 S \log y+C_{5}-2 n S \log 2 /(2-\delta) .
$$

As $n \rightarrow \infty$ this contradicts (56). We use the fact that the $O(1 / x)$ terms are insignificant if $x_{i}$ increase rapidly; e.g., if $\sum 1 / x_{i}<\infty$.

To obtain the second part of the theorem, let $\Lambda(u)$ oscillate about $y=+S$, as shown in Fig. 1, for $2 x_{i-1}<u<x_{i}-a$; near $y=-S$ for $x_{i}+a<u<2 x_{i}$; near $+S$ for $2 x_{i}<u<x_{i+1}-a$; and so on. By the default we mean, as above, the amount by which $\log \left|F\left(x_{n}\right)\right|$ falls short of the value it would have for an optimum $\Lambda(u)$, maximizing $F\left(x_{n}\right)$ (cf. Fig. 1). The same discussion as that given above to show that the default is unbounded, as $n \rightarrow \infty$, shows that the default tends to a finite limit when $n$ is fixed and $x_{n} \rightarrow \infty$. Suppose, now, that $x_{1}, \cdots, x_{n-1}$ have been chosen. With $c(x)$ the $c(x)$ in Theorem $\mathrm{XV}$, we choose $x_{n}$ so large that the default associated with $x_{1}, \cdots, x_{n-1}$ is less than $(1 / 2)\left|\log C\left(x_{n}\right)\right|$. The factor $1 / 2$ is used to absorb the constants and $O(1 / x)$ terms in our approximations. This gives the theorem. One could also estimate the maximum possible density of the $x_{n}$ 's in terms of $c(x)$, though we have not done so.

Extensions of the method. The following result is suggested by the preceding discussion:

Theorem XVI. Let $|\Lambda(u)-D u| \leqq H(u)$ where $H^{\prime \prime}(u) \leqq 0, \lim H(u)=\infty$, $\lim H^{\prime}(u)=0$. Then $\log |F(x)| \leqq G(x)$ where

$$
G(x) \sim 4 H(x) \log [x / H(x)]
$$


Moreover, there is a fixed function $F(x)$ and a sequence $x=x_{i} \rightarrow \infty$ such that $\log |F(x)|>G_{1}(x)$ where

$$
G_{1}(x) \sim 2 H(x) \log [x / H(x)] .
$$

Finally, there is a function $H(u)$ of the above type for which the first result is best possible, and also a function for which the second is best possible.

Since $(H / x) \log (x / H) \rightarrow 0$ when $H / x \rightarrow 0$, this result contains Theorem $\mathrm{I}$. For $H(u)$ we use a sufficiently small convex majorant of $|\Lambda(u)-D u|$. As a distinction between the two cases $H=$ constant, $H \rightarrow \infty$, we shall see later that the principal term in our present estimate is unaffected by the considerations leading to the distinction between XIV and XV; and of course the integral character of $\Lambda$ now plays no role.

A few trivial facts needed later are assembled here. First, $H^{\prime}(u)>0$. Thus, if $H^{\prime}\left(u_{0}\right)=0$, then $H^{\prime}(u) \leqq 0$ when $u>u_{0}$ by the condition on $H^{\prime \prime}(u)$; and this would make $\lim H(u)<\infty$. Second, we have $H(u)=o(u)$, since $\lim H^{\prime}(u)=0$, and therefore $H(u) / u \leqq C_{0}$ for some constant $C_{0}$. Finally, $H(u) / u$ is decreasing for large $u$. Thus,

$$
H(u)-H(\lambda)=(u-\lambda) H^{\prime}(\xi) \geqq(u-\lambda) H^{\prime}(u)
$$

so that $H(u) \geqq H(\lambda)+u H^{\prime}(u)-\lambda H^{\prime}(u)$. For large $u$, therefore, $H(u)>u H^{\prime}(u)$; and this shows that $[H(u) / u]^{\prime}<0$.

We turn now to the proof. The equation $D(x-u)=-H(u)$ determines a root $u$, which we designate as $x+\alpha$. By the mean value theorem

$$
H(x+\alpha)-H(x)=\alpha H^{\prime}(\xi), \quad x+\alpha>\xi>x .
$$

Since $D \alpha=H(x+\alpha)$ this yields

$$
\alpha=\frac{H(x)}{D-H^{\prime}(\xi)} \sim H(x) / D
$$

when we recall that $H^{\prime}(u) \rightarrow 0$. Proceeding in much the same way as before, we find that $\log |F(x)|$ is dominated, for a particular $x$, by

$$
\int_{\lambda}^{x-\alpha} H K+\int_{x-\alpha}^{x+\alpha} D(x-u) K-\int_{x+\alpha}^{\infty} H K .
$$

This result uses Lemma 4 of Theorem XIV, the role formerly taken by the lines $y= \pm H$ being now taken by the lines $y= \pm H(x+\alpha)$.

The third integral in (63) is dominated by

$$
\frac{H(x+\alpha)}{x+\alpha} \int_{x+\alpha}^{\infty} \frac{2 x^{2}}{u^{2}-x^{2}} d u \leqq H(x) \log [x / H(x)],
$$

since $H(u) / u$ is decreasing. (All our inequalities are asymptotic for $x \rightarrow \infty$ in a sense made explicit in Theorem XVI.) For the second integral (63) we have 


$$
\int_{x-\alpha}^{x+\alpha} D(x-u) K \sim 2 \alpha D \sim 2 H(x) .
$$

The first integral may be written $\int_{\lambda}^{s}+\int_{s}^{x-a}$, with $x-\alpha>s>\lambda$. We have

$$
\begin{gathered}
\int_{8}^{x-\alpha} H K \leqq H(x) \int_{8}^{x-\alpha} K \leqq H(x) \log \left[x^{3} /\left(H(x) s^{2}\right)\right], \\
\int_{\lambda}^{8} H K \leqq C_{0} \int_{\lambda}^{8} \frac{d u}{x^{2}-u^{2}} \leqq C x \log \frac{x+s}{x-s} \sim 2 C s
\end{gathered}
$$

where $C$ is constant, and $s=o(x)$ is assumed. Define $s=H(x)$. Then $s=o(x)$, and (67) is $o[H \log (x / H)]$. Also (66) is $H \log \left[x^{3} / H^{3}\right]$. Addition of (64)-(67) gives the first part of the theorem.

To prove the second part, we observe that

$$
\int_{\lambda}^{\log (x / H)} H K=o[H \log (x / H)], \quad \int_{x^{2}}^{\infty} H K=O(1)=o[H \log (x / H)],
$$

since $H(u) / u \leqq C_{0}$. The whole of the relevant contribution for a given $x$ comes, therefore, from the interval $\left(\log (x / H), x^{2}\right)$. For a suitable sequence $x_{i} \rightarrow \infty$ one can define the function at pleasure in each of these intervals without interference from the others (note that $\log (x / H) \rightarrow \infty$ ). Thus, the difficulty giving the distinction between XIV and XV does not arise here; we can get just as large an (asymptotic) value for log $|F(x)|$ with a single fixed function as with a function depending on $x$.

Let $A(u)=\Lambda(u)-D u$ follow the curve $y=H(u)$ from $u=\log (x / H)$ to the point where this curve meets the line $y=D(x-u)$; let it follow this line until it meets the curve $y=-H(u)$; and follow $-H(u)$ to $u=x^{2}$. The behavior before $\log (x / H)$ and after $x^{2}$ is a matter of no concern, provided $|\Lambda(u)-D u|$ $\leqq H(u)$. (When we say that $A(u)$ follows a given curve, we mean that it is within a distance 1 of the curve, and is dominated in absolute value by the curve.)

For this choice of $A(u)$ we have

$$
\int_{x+\alpha}^{x^{2}} A K \geqq A(x+\alpha) \int_{x+\alpha}^{x^{2}} K \geqq H(x) \log (H / x)
$$

using $H(x+\alpha)>H(x)$ and (62). If the curve $y=H(u)$ intersects $y=D(x-u)$ at $x-\beta$, we have $\beta<\alpha$ (also, $\beta$ satisfies (62)). Hence,

$$
\int_{\log (x / H)}^{x-\beta} \geqq \frac{H(x-\beta)}{x-\beta} \int \frac{2 x^{2}}{x^{2}-u^{2}} d u \geqq H(x) \log (x / H)
$$

the last inequality resulting from the fact that $\beta<\alpha \sim H / D$ and that $\log (x / H)=o(x)$. In view of (68) the proof of the second part of the theorem is complete. 
To establish the third part, concerning the optimum character of the results, we estimate the integral (66) differently, writing

$$
\int_{\lambda}^{s} H K \leqq \frac{2 x^{2}}{x^{2}-s^{2}} \int_{\lambda}^{s} H(u) d u / u,
$$

with asymptotic equality if $s=o(x)$. This yields

$$
\log |F(x)| \leqq 2 H(x) \log \frac{x^{2}}{s H(x)}+\frac{2 x^{2}}{x^{2}-s^{2}} \int_{\lambda}^{8} \frac{H(u)}{u} d u
$$

which is sometimes better than the first inequality of the theorem. For example if $H(u)=u^{a}, 0<a<1$, Equation (71) gives

$$
\log |F(x)| \leqq 2(1-a) x^{a} \log x,
$$

when we take $s=x / \log x$; the theorem gives twice this. It follows that the second inequality of the theorem is optimum for $H(u)=u^{a}$, since this second inequality then implies, asymptotically,

$$
\log |F(x)| \geqq 2(1-a) x^{a} \log x .
$$

Finally, the function $H(u)=\log \log u$ can be used to construct a function $F(x)$ such that, for $x=x_{i} \rightarrow \infty$, we have asymptotically

$$
\log |F(x)| \geqq 4 H(x) \log [x / H(x)] .
$$

This shows that the first inequality of the theorem is also optimum. To prove (74), write

$$
\int_{p}^{x-\beta} H K=\int_{p}^{8}+\int_{8}^{x-\beta}
$$

where $p=\log (x / H)$ and $s>p, s=o(x)$; we choose $s=x^{1 / 2}$ for our present purposes. When $x$ is large,

$$
\int_{p}^{8} H K \sim 2 \int_{p}^{8} H(u) d u / u
$$

and

$$
\int_{8}^{x-\beta} H K \geqq H(x-\beta) \log (x / \beta) .
$$

With the given choice of $H(u), p$, and $s$, Equation (75) gives $2 \log x \log \log x$, and (76) gives $\log x \log \log x$. Combining with the term $\int_{x+\alpha}^{x^{2}}$ yields (74), when we construct a suitable sequence $x_{i} \rightarrow \infty$. If we had chosen $H(u)=\log u$, the same procedure would show that the precise (that is, optimum) inequality is 


$$
\log |F(x)| \leqq 3 H \log (x / H)
$$

Completeness on an open interval. Using a method given in [2], Levinson [3] has established the following theorem:

Theorem A (Paley, Wiener, And Levinson). Let $\phi(x)$ be an even, positive, increasing function of $|x|$ such that $\int_{1}^{\infty}\left[\phi(x) / x^{2}\right] d x<\infty$. If $\delta>0$, then there exists an entire function $H(x) \not \equiv$ such that $|H(x)| \leqq e^{-\phi(x)}$ and such that $h(u)$, the Fourier transform of $H(z)$, vanishes outside of $|u| \leqq \delta$.

From this we deduce

THEOREM XVII. Let $F(z)$ be an entire function of type $T$, with real or complex zeros $\lambda_{n}$, and let $\phi(x)$ satisfy the requirement of Theorem A. Suppose there exists an entire function $G(z)$, of type $<T^{\prime}$, such that $|F(x) G(x)|<e^{\phi(x)}$. Then the set $\left\{e^{i \lambda_{n} x}\right\}$ is incomplete $L^{2}$ on some interval of length $<T+T^{\prime}$.

A partial converse is also true. If the set is incomplete $L^{2}$ on an interval of length $<T+T^{\prime}$, then there is an entire function $G(x)$, of type $<T^{\prime}$, such that $\int_{1}^{\infty}\left(|\log | F(x) G(x)|| / x^{2}\right) d x<\infty$.

LEмма. In Theorem XVII, there exists an entire function $H(z)$, of type $<T^{\prime}$, such that $|F(x) H(x)|<e^{-\phi(x)}$.

A trivial consequence of Theorem A, this lemma has the curious property that the hypothesis becomes weaker, and the conclusion stronger, the larger we take $\phi(x)$. To prove it, let the type of $G(z)$ be $T^{\prime}-2 \delta$ in Theorem XVII, where $\delta>0$. Without loss of generality we may assume $\phi(x)$ in Theorem $\mathrm{A}$ so large that $H(x) \in L^{2}$. Hence $H(x)$ is the inverse Fourier transform of its transform, so that $H(z)$ is representable as

$$
H(z)=\int_{-\delta}^{\delta} h(x) e^{i z x} d x .
$$

Choose $2 \phi(x)$ instead of $\phi(x)$ in Theorem A, so that $H(z)$ has type $\leqq \delta$ by (77) and satisfies $|H(x)|<e^{-2 \phi(x)}$. Therefore $G H$ is of type $<\left(T^{\prime}-2 \delta\right)+\delta$ $<T^{\prime}$, and $|(G H) F|<e^{\phi} e^{-2 \phi}=e^{-\phi}$.

To obtain XVII, choose $\phi(x)$ larger (if necessary) so that $\int_{0}^{\infty} e^{-2 \phi(x)} d x<\infty$. Then there is a function $G(z)$ for which $F G \in L^{2}$ and for which $F G$ has type $\leqq T+T^{\prime}-\delta$. By a theorem of Paley and Wiener $[2 ; 6]$ we can represent $F G$ as

$$
F(z) G(z)=\int_{-\left(T+T^{\prime}-\delta\right)}^{T+T^{\prime}-\delta} f(x) e^{i z x} d x, \quad f(x) \in L^{2} .
$$

Since $F\left(\lambda_{n}\right) G\left(\lambda_{n}\right)=0$, the set $\left\{e^{i \lambda_{n} x}\right\}$ is not complete on an interval of length $T+T^{\prime}-\delta$.

If $\left\{\lambda_{n}\right\}$ is a real, positive increasing sequence, and $\Lambda(u)=$ number $\lambda \leqq u$, 
Levinson [1] proved that $\left\{e^{i \lambda_{n} x}\right\}$ is complete on every interval of length $<2 \pi D$ when

$$
\limsup _{y \rightarrow 1+} \limsup _{x \rightarrow \infty} \frac{\Lambda(x y)-\Lambda(x)}{x y-x}=D .
$$

Later it was shown [3] that $\left\{e^{ \pm i \lambda_{n} x}\right\}$ is not complete on any interval of length $>2 \pi D$ if

$$
\limsup _{y \rightarrow \infty} \limsup _{x \rightarrow \infty} \frac{\Lambda(x+y)-\Lambda(x)}{(x+y)-x}=D .
$$

We are in a position, now, partially to close the gap between these two results.

TheOREM XVIII. Let $\left\{\lambda_{n}\right\}$ be a positive increasing sequence, and $\Lambda(u)$ the number of $\lambda_{n}<u$. If

$$
\limsup _{x \rightarrow \infty} \frac{\Lambda(x+x y)-\Lambda(x)}{(x+x y)-x}=D
$$

for a positive function $y(x)$ such that $y^{\prime} \leqq 0,(x y)^{\prime} \geqq 0, \int_{1}^{\infty} y \log y d x / x$ converges, then $\left\{e^{ \pm i \lambda_{n} x}\right\}$ is not complete on any interval of length greater than $2 \pi D$.

The proof depends on the following:

LEммA. If $|\Lambda(u)-D u|<u y(u)$ for a function $y(u)$ satisfying the requirements of Theorem XVIII, then $\left\{e^{ \pm i \lambda_{n} x}\right\}$ is not complete on any interval of length exceeding $2 \pi D$.

For proof, let $F(z)=\prod\left(1-z^{2} / \lambda_{n}^{2}\right)$, as usual. With $H(u)=u y(u)$, the hypothesis implies that $H / u$ is decreasing and $H(u)$ increasing, so that XVI applies. Since $\int_{1}^{\infty} H(u) \log (u / H) d u / u^{2}<\infty$, the lemma follows from XVII. To obtain XVII, let $D^{\prime}=D+\epsilon$ with $\epsilon>0$. Form a sequence of points $\left\{x_{n}\right\}$, defined recursively by

$$
x_{n+1}=x_{n}+x_{n} y\left(x_{n}\right)
$$

If $x_{1}$ is large enough,

$$
\Lambda\left(x_{n+1}\right)-\Lambda\left(x_{n}\right)<D^{\prime} x_{n}
$$

for $n=1,2,3, \cdots$. Add enough zeros in $\left(x_{1}, x_{2}\right)$ so that (within \pm 1 , say) $\Lambda\left(x_{2}\right)=D^{\prime} x_{2}$. Equation (78) shows that $\Lambda\left(x_{3}\right)<D^{\prime} x_{3}$. Add enough zeros in $\left(x_{2}, x_{3}\right)$ to make $\Lambda\left(x_{3}\right)=D^{\prime} x_{3}$, and so on. Thus, we can adjoin a sequence $\lambda^{*}$ so that for the new function, $\Lambda^{*}$, we have $\Lambda^{*}\left(x_{n}\right)=D^{\prime} x_{n}$ within \pm 1 . Since $\Lambda^{*}(u)$ is increasing

$$
\left|\Lambda^{*}(u)-D^{\prime} u\right|<D^{\prime}\left(x_{n+1}-x_{n}\right)=D^{\prime} x_{n} y\left(x_{n}\right)<D^{\prime} u y(u)
$$

when $x_{n}<u<x_{n+1}$, hence for all large $u$. Hence the enlarged set $\left\{\lambda, \lambda^{*}\right\}$ is 
not complete on an interval of length greater than $2 \pi D^{\prime}$, by the lemma.

It is worth mentioning that the restriction to real $\lambda_{n}$ plays an essential role in the character of our theorems. If complex $\lambda$ 's are allowed, one can give other criteria for completeness. For example, let $\left\{\lambda_{n}\right\}$ be a set of complex numbers such that

$$
\sum \frac{\left|\operatorname{Im}\left(\lambda_{n}\right)\right|}{\left|\lambda_{n}\right|^{2}}=\infty
$$

Then $\left\{e^{i \lambda_{n} x}\right\}$ is complete $L^{2}$ on every finite interval. This is an immediate consequence of the work of Levinson.

Sets with finite excess. Paley and Wiener [2] say that a set $\left\{e^{i \lambda_{n} x}\right\}$ has deficiency $d$ on a given interval if the set becomes complete when $d$ but not fewer functions $\left\{e^{i \alpha x}\right\}$ are added to the set. An excess $e$ is similarly defined, and the set is said to be exact if $d=0$.

THEOREM XIX. Let $\left\{\lambda_{n}\right\}$ be a nondecreasing sequence of positive real numbers with $\Lambda(u)$ the number of $\lambda^{\prime} s \leqq u$. If $|\Lambda(u)-D u| \leqq H$, then the excess of the set $\left\{e^{ \pm i \lambda_{n} x}\right\}$ on an interval of length $2 \pi D$ does not exceed $4 H-3 / 2$. Moreover, there is a distribution $\Lambda(u)$ satisfying $|\Lambda(u)-D u| \leqq H$ with excess as large as [4H-2] or, if $4 H-2$ is an integer, as large as $4 H-3$.

The first part of the theorem improves, and the second completes, a theorem of Paley and Wiener. With the results of Levinson, this particular question concerning completeness is practically settled. He shows that the deficiency is sometimes as large as $[2 A-3 / 2-\delta]$, and never as large as $2 A-3 / 2$. We show that the excess is sometimes as large as [4H-2] or $4 H-3$, and never larger than $4 H-3 / 2$.

To prove the result, let $F(z)=\prod\left(1-z^{2} / \lambda_{n}^{2}\right)$ as usual, and let $N$ be an integer with $N=4 H-3 / 2+\delta, \delta>0$. To remove $N$ terms $\left\{e^{i \lambda_{n} x}\right\}$ is to divide $F(x)$ by a polynomial $P(x)$ of degree $N$. Theorem $\mathrm{XV}$ yields

$$
G(x)=F(x) / P(x)=o\left(x^{-1 / 2-\delta}\right) .
$$

Also lim sup $\log |G(z)| /|z|=\pi D$, since $\Lambda(u) \sim D u$. Using a theorem of Paley and Wiener [2] (as extended by Plancherel and Pólya [6]) we can write

$$
G(x)=\int_{-\pi D}^{\pi D} g(x) e^{i z x} d x, \quad g(x) \in L^{2} .
$$

Hence the curtailed sequence $\left\{e^{i \lambda_{n} x}\right\}$ is not complete.

To prove the second part of the theorem, with $\delta=4 \mathrm{H}-2-[4 \mathrm{H}-2]>0$ let $\lambda_{n}$ be a sequence satisfying $|\Lambda(u)-D u| \leqq H$ and such that

$$
F(x)>x^{4 H-2-\delta}
$$

for an infinite sequence $x=x_{i} \rightarrow \infty$. That such an $F(x)$ exists follows from Theorem XV with $C(x)=x^{-\delta}$. To remove $[4 H-2] \lambda^{\prime}$ s is to form 


$$
G(x)=F(x) / P(x),
$$

where $P(x)$ is a polynomial of degree $[4 H-2]$. For $x=x_{i}$ we have

$$
G(x)>x^{4 H-2-\delta} x^{-[4 H-2]}=1 .
$$

Hence $\int_{-\infty}^{\infty}|G(x)|^{2} d x=\infty$; in the contrary case we should have

$$
G(x)=\int_{-\pi D}^{\pi D} e^{i z x} g(x) d x, \quad g(x) \in L^{2},
$$

and this entails $G(x) \rightarrow 0$ by the Riemann-Lebesgue lemma (cf. [6]). The curtailed set $\left\{e^{i \lambda_{n} x}\right\}$ is therefore complete [1].

\section{REFERENCES}

1. N. Levinson, Gap and density theorems, Amer. Math. Soc. Colloquium Publications, vol. 26, 1940, pp. 6, 13, 25 ff., 81, 89 ff., 244.

2. R. E. A. C. Paley and N. Wiener, Fourier transforms, Amer. Math. Soc. Colloquium Publications, vol. 19, 1934, pp. 13, 68 ff., $86 \mathrm{ff}$.

3. R. M. Redheffer, Remarks on incompleteness of $\left\{e^{i \lambda_{n} x}\right\}$, nonaveraging sets, and entire functions, Proc. Amer. Math. Soc. vol. 2 (1951) pp. 365-369.

4. F. Carlson, Über Potenzreihen mit endlich vielen verschiedenen Koeffizienten, Math. Ann. vol. 79 (1919) pp. 237-245.

5. E. C. Titchmarsh, The theory of functions, Oxford University Press, 1939, pp. 183-184.

6. M. Plancherel and G. Poblya, Fonctions entières et intégrales de Fourier multiples, Comment. Math. Helv. vol. 9 (1936-37) pp. 224-248.

7. A. Pfluger, Ueber gewisse ganze Funktionen vom Exponentialtypus, Comment. Math. Helv. vol. 16 (1943) pp. 1-18.

8. E. C. Titchmarsh, On integral functions with real negative zeros, Proc. London Math. Soc. (2) vol. 26 (1927) pp. 185-200.

9. N. A. Bowen, $A$ function-theory proof of Tauberian theorems on integral functions, Quart. J. Math. vol. 19 (1948) pp. 90-100.

10. M. E. Noble, Extensions and applications of a Tauberian theorem due to Valiron, Proc. Cambridge Philos. Soc. vol. 47 (1951) pp. 22-37.

11. B. Levin, On functions of finite degree, bounded on a sequence of points, Doklady Akad. Nauk. SSSR. vol. 65 (1949) pp. 265-268.

12. S. Mandelbrojt, Séries adherantes, 1952, pp. $62 \mathrm{ff}$.

13. N. A. Bowen and A. J. Macintyre, An oscillation theorem of Tauberian type, Quart. J. Math. Oxford Ser. (2) vol. 1 (1950) pp. 243-247.

\section{UNIVERSITY OF CALIFORNIA,} Los Angeles, Calif. 